\title{
On the criteria guiding the design of the upper electron-cyclotron launcher for ITER
}

\author{
E. Poli ${ }^{1}$, C. Angioni ${ }^{1}$, F. J. Casson ${ }^{1}$, D. Farina ${ }^{2}$, L. Figini' ${ }^{2}$, T. P. Goodman ${ }^{3}$, O. Maj ${ }^{1}$, O. Sauter ${ }^{3}$, H. Weber ${ }^{1}$, H. Zohm ${ }^{1}$, \\ G. Saibene ${ }^{4}$, and M. A. Henderson ${ }^{5}$ \\ ${ }^{1}$ Max-Planck-Institut für Plasmaphysik, Garching, Germany \\ ${ }^{2}$ Istituto di Fisica del Plasma CNR, EURATOM-ENEA-CNR Association, Milano, Italy \\ ${ }^{3}$ Centre de Recherches en Physique des Plasmas, CRPP-EPFL, Lausanne, Switzerland \\ ${ }^{4}$ Fusion for Energy, Barcelona, Spain \\ ${ }^{5}$ ITER Organization, Saint-Paul-Lez-Durance, France
}

\begin{abstract}
Electron cyclotron waves injected from an antenna located in the upper part of the vessel will be employed in ITER to control MHD instabilities, particularly neoclassical tearing modes (NTMs). The derivation of the NTM stabilization criteria used up to now to guide the optimization of the launcher is reviewed in this paper and their range of validity elucidated. Possible effects leading to a deterioration of the predicted performance through a broadening of the EC deposition profile are discussed. The most detrimental effect will likely be the scattering of the EC beams from density fluctuations, resulting in a beam broadening in the $100 \%$ range. The combined impact of these effects with that of beam misalignment (with respect to the targeted surface) is discussed for a time slice of the standard $Q=10 \mathrm{H}$-mode scenario.
\end{abstract}

\section{Introduction}

The ITER upper launcher (UL) for electron cyclotron (EC) waves is presently under development in the frame of a European collaboration and has reached recently the preliminary design level [1]. Its goal is the control of magnetohydrodynamical instabilities, in particular Neoclassical Tearing Modes (NTMs). A maximum power of $20 \mathrm{MW}$ (at least in the first operation phase, on account of transmission losses) will reach the plasma through four ports, each containing eight beam lines divided in two rows. The four beams of each row will be steered through independent steering mirrors, referred to as the upper steering mirror (USM) and lower steering mirror (LSM). In this configuration, the power which can be injected from each row amounts to $13.3 \mathrm{MW}$.

The development of the UL requires a continuous check that the required physical performance is achieved without violating the technical constraints set by the ITER environment. Criteria that allow a simple evaluation of the launcher performance with respect to NTM stabilization are extremely useful, also because their inspection provides an indication of the quantities that should be optimized by the system. Such stabilization criteria for ITER parameters have been provided by Zohm et al. [2] and Sauter et al. [3] (later confirmed by Bertelli et al. [4]). Zohm's criterion states that NTM stabilization is achieved if the driven current density is larger by a factor 1.2 than the unperturbed bootstrap current density at the relevant magnetic surface. This criterion was derived assuming that the width of the EC deposition profile will be much larger than the marginal island size at which the tearing mode self-stabilizes. Moreover, it requires that the EC power is modulated, so that the current is driven only around the O-point of the island. On the basis of this criterion, the toroidal launch angle $\beta$ for the UL was fixed in the present design to $\beta=20^{\circ}$. According to Sauter's criterion, on the other hand, the total driven current should be optimized, if the CD profile width can be kept below a threshold, found to be $5 \mathrm{~cm}$ for ITER. As a matter of fact, beam tracing calculations performed for the last available version of the UL design and of the ITER plasma parameters [5] predict narrow deposition widths (in the range of $3-5 \mathrm{~cm}$ or even below), which means that the beams deposit their power well inside the island, at least for most of the stabilization process (the marginal island size in ITER is not predicted with certainty by the present theories), and there is no advantage deriving from further focusing. Other than in the case of large deposition widths considered in Zohm's criterion, for narrow deposition profiles continuous wave (cw) injection exhibits a stabilization efficiency just about $20 \%$ lower than that of modulated injection.

However, there is a variety of physical processes which have been studied in the literature that could lead to a broadening (with respect to standard beam tracing calculations) of the deposition profile, thus lowering the peak current density for a given total driven current. In particular, radial transport of EC-heated electrons [6] and beam scattering from density fluctuations [7] could lead to broadening in the $100 \%$ range. In the frame of the evaluation of 
the UL performance, the impact of beam broadening has to be taken into account. As emerges from the previous discussion, profile broadening will increase significantly the stabilization power when a significant fraction of the current is driven outside the island. In this case, modulation could become necessary.

In this paper, a common derivation of the stabilization criteria described above is presented, which helps clarify their validity and the respective applicability range. Possible reductions of the peak current density are discussed and estimates for the power requirements for NTM stabilization for a selected ITER equilibrium are presented.

\section{Derivation of the stabilization criteria}

The first step of the derivation of simple stabilization criteria for the neoclassical tearing mode in ITER is to simplify the generalized Rutherford equation $[8,9]$, retaining only those terms that are believed to lead to the largest contribution to the NTM dynamics [2-4], namely those involving the classical stability parameter $\Delta^{\prime}$, the bootstrap current destabilization and the effect of the helical component of the EC current on the island evolution.

As mentioned in Sec. 1, the first simple quantitative criterion for NTM suppression used to assess the performance of the UL was formulated as [2]

$$
\eta_{N T M} \equiv \frac{j_{\mathrm{CD}}}{j_{\mathrm{bs}}}>1.2,
$$

where $j_{\mathrm{CD}}$ and $j_{\mathrm{bs}}$ are the peak EC-driven current density and the (unperturbed) bootstrap current density at the surface of interest, respectively. Successively, it was proposed to employ a different criterion, namely [3, 4]

$$
\eta_{N T M} w_{\mathrm{CD}}>5 \mathrm{~cm}, \quad w_{\mathrm{CD}}<5 \mathrm{~cm},
$$

where $w_{\mathrm{CD}}$ is the full width of the ECCD profile. In the following, a common derivation of both Eqs.(1) and (2) is presented. It closely follows the discussion of Ref. [2].

The condition for a stable island size $\mathrm{d} w / \mathrm{d} t=0(w$ is the island full width) can be written as

$$
0=-1+\frac{w_{\mathrm{sat}}}{w}-5 \frac{w_{\mathrm{CD}} w_{\mathrm{sat}}}{w^{2}} \eta_{N T M} \eta_{\mathrm{CD}},
$$

where the saturated island width $w_{\text {sat }}$ expresses the balance between the neoclassical (bootstrap) drive and the stabilization due to the equilibrium current profile in the absence of ECCD, and the CD stabilization efficiency $\eta_{\mathrm{CD}}$ is defined as in Refs. [10,11]. Requiring that no roots of Eq. (3) exist leads to the following criterion for NTM stabilization:

$$
\frac{w_{\mathrm{CD}}}{w_{\mathrm{sat}}} \frac{j_{\mathrm{CD}}}{j_{\mathrm{bs}}} \eta_{\mathrm{CD}}>\frac{1}{20} .
$$

We can now distinguish between the case in which the ECCD profile is narrower or broader than the typical island width $w_{\text {marg }}$ at which stabilization occurs. In the former limit, $w_{\mathrm{CD}}<w_{\text {marg }}$, the stabilization efficiency is approximately $\eta_{\mathrm{CD}} \lesssim 0.4$ for both continuous injection and $50 \%$-duty-cycle modulation. Eq.(4) then becomes

$$
\frac{j_{\mathrm{CD}}}{j_{\mathrm{bs}}} \frac{w_{\mathrm{CD}}}{w_{\mathrm{sat}}}>\frac{1}{8} \text {. }
$$

For a saturated island width of the order of $32 \mathrm{~cm}$, as estimated for the $q=2$ surface in ITER [3], this condition yields

$$
\eta_{N T M} w_{\mathrm{CD}} \gtrsim 4 \mathrm{~cm},
$$

which is in qualitative and even reasonable quantitative agreement with Sauter's stabilization criterion, derived in Ref. [3] and confirmed in Ref. [4] through a more detailed analysis than the simple calculation presented here. It should be stressed that our analytic derivation allows not only a clarification of the limit of validity of Eq. (2), but also its dimensionless reformulation and hence its application for instance to the case of different $w_{\text {sat }}$, e. g. at the $q=3 / 2$ surface in ITER.

In the opposite limit, $w_{\mathrm{CD}}>w_{\text {marg }}$, the $\mathrm{CD}$ stabilization efficiency can be approximated as $\eta_{\mathrm{CD}} \simeq 0.15 w / w_{\mathrm{CD}}$ for modulated injection in the O-point ( $50 \%$ duty cycle) and $\eta_{\mathrm{CD}} \simeq w^{2} / 8 w_{\mathrm{CD}}^{2}$ for continuous injection [2]. To express the stabilization condition it is more practical to exploit the fact that in ITER it can be assumed that $w_{\text {marg }}$ is much smaller than $w_{\text {sat }}$, so that the first term on the righthand side of Eq.(3) can be dropped near marginal stability. In case of modulated injection, Eq. (3) leads then directly to the result that stabilization is achieved if $\eta_{N T M}>4 / 3$. This condition is very similar to Zohm's criterion given above (1), which includes a reduction of the neoclassical drive due to geodesic-curvature effects [12]. In a similar way, one can show that, in the limit of large deposition width and for continuous injection, complete stabilization $(w \rightarrow 0)$ can be achieved only with an infinite ECCD current (no effects leading to stability of small islands are considered in the above derivation). In other words, for large CD profiles, power modulation becomes essential for NTM suppression.

An obvious limitation of the previous analysis is the fact that $w_{\text {marg }}$ had to be introduced ad hoc in order to obtain an estimate of the CD stabilization efficiency $\eta_{\mathrm{CD}}$ in the corresponding limit, whereas $w_{\text {marg }}$ should appear in Eq. (3) from the beginning. Moreover, the stabilization efficiency $\eta_{\mathrm{CD}}$ should be known for arbitrary values of $w / w_{\mathrm{CD}}$. Eq. (3) is then reformulated as

$$
0=-1+\frac{w_{\mathrm{sat}} w}{w^{2}+w_{\mathrm{marg}}^{2}}-5 \frac{w_{\mathrm{CD}} w_{\mathrm{sat}}}{w^{2}} \frac{j_{\mathrm{CD}}}{j_{\mathrm{bs}}} \eta_{\mathrm{CD}}\left(w / w_{\mathrm{CD}}\right),
$$

including the incomplete pressure flattening inside the island due to finite perpendicular transport [13] as a stabilizing mechanism for small islands. Our incomplete knowledge of the physics determining the stability of small islands is taken into account by considering $w_{\text {marg }}$ as a parameter, which will be varied in the following between 1 and $6 \mathrm{~cm}$. For $\eta_{\mathrm{CD}}$, the fits reported in Ref. [14] are used.

The value of $\eta_{N T M}$ corresponding to unconditional stability, i. e. the value at which the right-hand side of Eq.(6) becomes negative for all values of the island width $w$, can be plotted for different values of $w_{\mathrm{CD}}$ to check the validity of the criteria derived above. In Fig. 1, the saturated island width is imposed to be $32 \mathrm{~cm}$. For large CD deposition widths the value of $\eta_{N T M}$ required for stabilization saturates to a constant level smaller than 1.2 if modulation is 

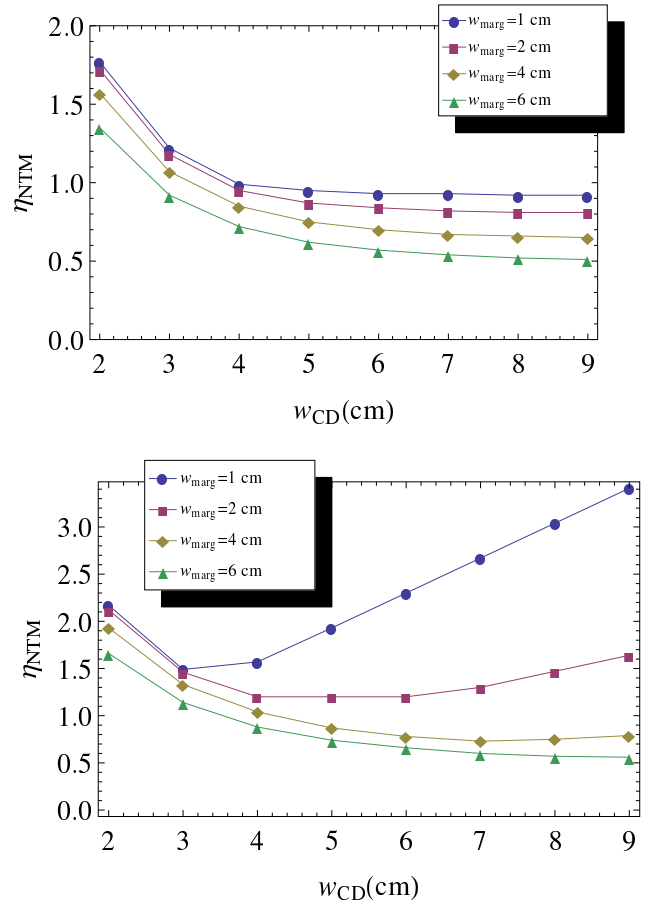

Figure 1. The value of $\eta_{N T M}$ corresponding to unconditional stability as a function of the width of the ECCD profile for different values of $w_{\text {marg }}$ and for $w_{\text {sat }}=32 \mathrm{~cm}$. The upper plot corresponds to modulated ECCD, the right plot to continuous wave injection.
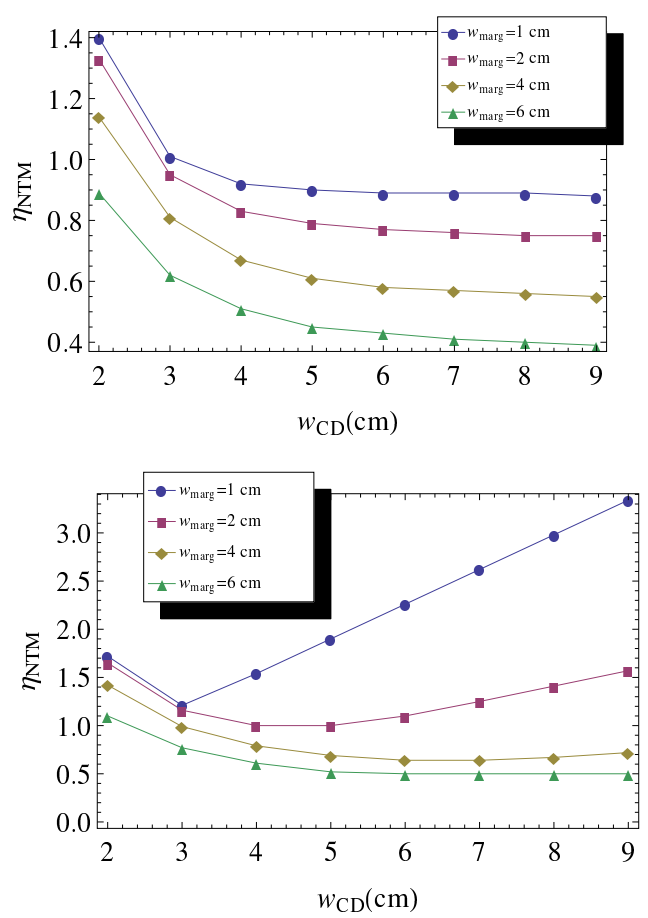

Figure 2. The same as Fig. 1 for the $q=3 / 2$ surface.

included, while it increases with $w_{\mathrm{CD}}$ for continuous injection. For small deposition profiles, on the other hand, modulation becomes unessential and $\eta_{N T M}$ scales like $1 / w_{\mathrm{CD}}$, i. e. $w_{\mathrm{CD}} \times \eta_{N T M}$ approaches a constant. For the $q=3 / 2$ surface, the estimated value of $w_{\text {sat }}$ is smaller $(25 \mathrm{~cm})$ and the requirements for NTM stabilization are less stringent than for the $q=2$ surface, see Fig. 2.
The previous figures suggest that the existing stabilization criteria are valid also for a "worst-case" scenario in which the marginal island width is as small as 1 centimetre. In this sense they are conservative, representing rather a sufficient than a necessary condition for NTM suppression. It is finally noted that Eq. (1) and Eq. (2) lead to the same value of $\eta_{N T M}$ for NTM suppression for $w_{\mathrm{CD}}=w_{\mathrm{CD} \text {, crit }}=5 / 1.2 \simeq 4.2 \mathrm{~cm}$. For $w<w_{\mathrm{CD} \text {, crit }}$, Sauter's criterion (2) is to be employed, while Zohm's criterion (1) applies at larger $w_{\mathrm{CD}}$. Since each criterion leads to stronger requirements on $\eta_{N T M}$ (and hence on the required injected power) in its respective domain of validity, it can be inferred that $w_{\mathrm{CD} \text {,crit }}$ represents a sort of optimum deposition width, corresponding to the best compromise between localization and maximization of the driven current. Beam tracing calculations performed for simulated ITER discharges show that the present launcher setup allows very narrow deposition profiles [5], with deposition widths below $3 \mathrm{~cm}$ for beams injected from the LSM onto the $q=2$ surface. For this reason, it has been proposed to increase the toroidal injection angle to increase the driven current and approach the "ideal" width $w_{\mathrm{CD} \text {,crit }}[4]$.

\section{Broadening of the EC deposition profiles}

Ray/beam tracing modelling employed to determine the ECCD profiles in ITER relies on the solution of the equations of geometrical optics or diffractive beam tracing, coupled to linear or quasilinear modelling of absorption and current drive [15]. Below, the validity of some assumptions typical of this "standard" approach are addressed, with particular emphasis on those effects which can potentially modify significantly the narrow deposition profiles mentioned above.

A first common approximation in the calculation of ECCD for NTM stabilization is to neglect the presence of the magnetic island. Recently, it has been observed that wave absorption close to the O-point of an island, resulting in the deposition of the power on a narrow flux tube, could lead to significant power densities and thus to deviation from linear calculations. Specifically, reduced absorption due to quasi-linear flattening of the distribution function could lead to a shift of the power deposition towards regions of lower CD efficiency. This effect has been calculated for present day machines and found to be small [16]. It should be negligible for ITER, due to its larger size. A second approximation results from taking a single "representative" beam for each mirror and multiplying the result by the number of beams (four) for each mirror, i. e. from assuming perfect superposition of the beams, independently from the plasma parameters and the steering angle. The broadening (with consequent peak reduction) due to incomplete beam overlap must still be quantified, but previous studies indicate that it should be possible to keep deviations from the ideal single-beam profiles below $10 \%$ [17]. Aberration effects, i. e. deviations from the paraxial approximation, have been investigated using a new quasi-optical code for ITER-like scenarios. The 


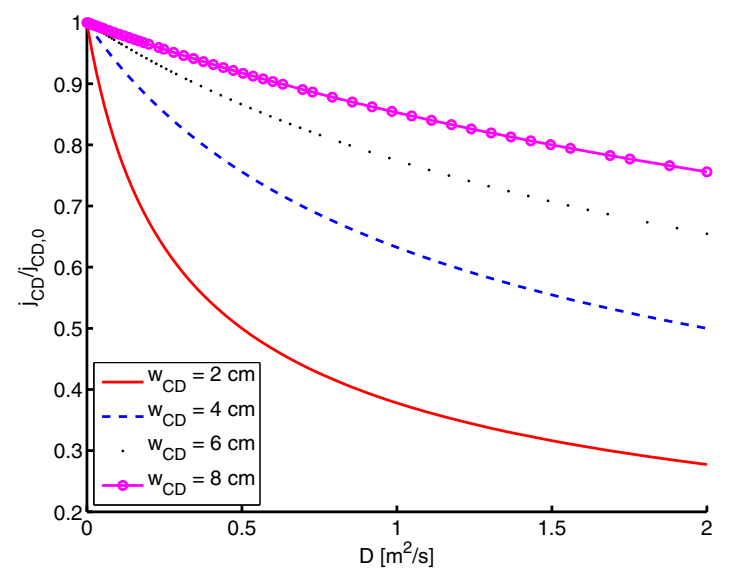

Figure 3. Reduction of the $j_{\mathrm{CD}}$ peak due to radial transport as a function of the diffusion coefficient according to Eq. (7) for $\tau=$ $6 \cdot 10^{-4} \mathrm{~s}$ (typical of electrons at 2.5 times the thermal velocity for ITER parameters at the $q=2$ surface).

broadening of the EC profiles due to aberration has been found in the range between 10-20\% [18]. The broadening occurs when dispersive absorption causes the damping of different wave vectors at different locations [19]. A simplified model used in Ref. [19] has confirmed that beam broadening due to aberration is in the range of $\lesssim 10 \%$.

While the effects mentioned above lead to modest modifications of the ECCD profiles (considering the high localization predicted by beam tracing calculations), there are other processes whose impact is potentially much more detrimental. Small deviations of the beam path caused by fluctuations of the medium (typically of its density) can have a sizeable effect in reactor-size plasmas, where the waves propagate over a large distance after deflection. Recent attempts at quantifying scattering-induced beam broadening, based on a Fokker-Planck modelling of the diffusion of rays [7] or on ray tracing in the presence of fluctuations that satisfy WKB ordering [20] suggest an increase of the beam width at the absorption layer in ITER of the order of $100 \%$. Preliminary results obtained with a more sophisticated approach, based on the solution of the wave kinetic equation in the presence of small-scale fluctuations retaining diffraction, full tokamak geometry and determination of the power absorption profile, and implemented in the wave-kinetic-equation solver WKBeam, have found a broadening of the same order for ITER parameters [21]. In all these studies, turbulence is modelled as a layer of density fluctuations with $\delta n_{e} / n_{e}$ in the $10 \%$ range located at the plasma periphery. According to our present understanding, this effect will lead to the most severe loss of localization of the EC deposition profiles for NTM stabilization in ITER, with broadening of the beam by about a factor of two (and consequent reduction of the peak current drive by the same factor). More detailed studies will be needed to achieve better quantitative predictions.

A second effect potentially leading to a broadening of the CD profile of comparable size arises from the fact that in a reactor the time scale on which the current drive pro- file arising from the Fisch-Boozer effect establishes can be comparable with the time on which the heated electrons are transported away from the region where the power is absorbed. An analytic formula was proposed in Ref. [6] to determine the reduction of $j_{\mathrm{CD}}$ in the presence of transport:

$$
\frac{j_{\mathrm{CD}}}{j_{\mathrm{CD}, 0}}=\frac{w_{\mathrm{CD}}}{\sqrt{w_{\mathrm{CD}}^{2}+4 D \tau}},
$$

where $j_{\mathrm{CD}, 0}$ and $w_{\mathrm{CD}}$ are the peak value of the driven current density and the full width of the CD profile in the absence of transport, while $D$ and $\tau$ are the diffusion coefficient and the collision time of the current-carrying electrons, respectively. The value of $j_{\mathrm{CD}} / j_{\mathrm{CD}, 0}$ from Eq. (7) is plotted against $D$ in Fig. 3 for different values of $w_{\mathrm{CD}}$. It is clear that the effect is particularly pronounced when the $\mathrm{CD}$ profile is narrow and the transport coefficient is high. For CD profiles in the range of 3-4 cm, and a diffusion coefficient of $1 \mathrm{~m}^{2} / \mathrm{s}$, the peak value of $j_{\mathrm{CD}}$ is reduced by a factor of two. Gyrokinetic simulations of the turbulent transport for parameters typical of the $q=3 / 2$ and $q=2$ surfaces in ITER have been performed, with the goal of determining some constraints on the range of possible values attained by the diffusion coefficient $D$ in the region of velocity space affected by the interaction with the EC beam [22]. These simulations indicate relatively low values of $D$, in the range of $0.1-0.2 \mathrm{~m}^{2} / \mathrm{s}$, for passing electrons with a velocity between 1.5 and 2.5 times the thermal velocity (it should be noted that these simulations do not include the magnetic island, inside which the gradients driving the turbulence are actually reduced). These low values of the diffusion coefficient, in combination with the beam broadening due to scattering from density fluctuations discussed above, points to a relatively small role (again at the $10 \%$ level) of transport effects on the determination of the CD profiles for the UL in ITER.

\section{Impact on the power requirements for NTM suppression in ITER}

An increase of the $\mathrm{CD}$ profile width at constant total driven current is not automatically detrimental for NTM suppression, as long as the current is driven well inside the island (in this case, the quantity that should be maximized is indeed the total driven current, see Sec. 2). Whether this is the case during the stabilization process, depends mainly on the marginal island size, whose value is known only with a large uncertainty. We investigate here the consequences of profile broadening on the power required for NTM suppression solving Eq. (6) varying $w_{\mathrm{CD}}$ and $j_{\mathrm{CD}}$ so that their product remains constant and equal to the value calculated by the TORBEAM code [23]. In the helical efficiency $\eta_{C D}$, the effect of a possible misalignment between the resonant surface and the position of maximum power deposition is included. For islands of the size of the CD profile width $w_{\mathrm{CD}}$, a misalignment of the order of half $w_{\mathrm{CD}}$ leads to a loss of the stabilizing effect of ECCD. The present steering accuracy envisaged for the UL, $\Delta \alpha=0.1^{\circ}$, should be sufficient to compensate for such a misalignment 


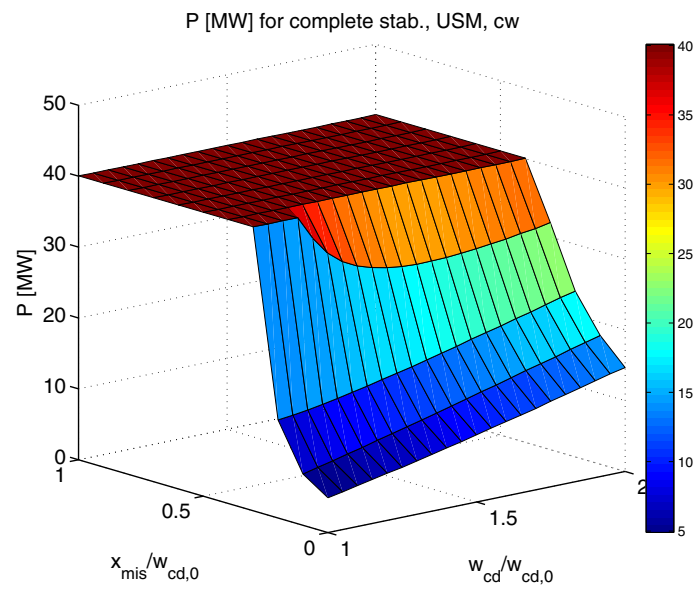

$\mathrm{P}[\mathrm{MW}]$ for complete stab., LSM, cW

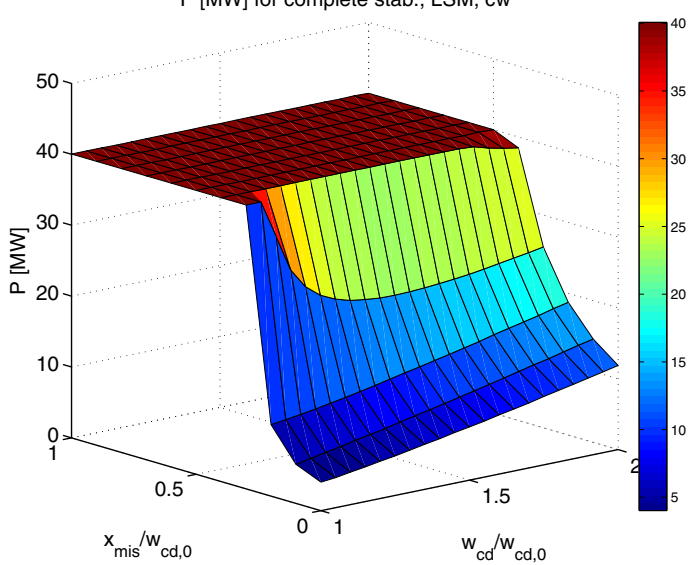

Figure 4. Power requirements for complete NTM suppression as a function of the profile broadening and of the misalignment for the $q=3 / 2$ surface. The upper figure refers to the USM, the lower one to the LSM. CW injection is considered, with $w_{\text {marg }}=$ $2 \mathrm{~cm}$.

(cf. the estimates presented in Ref. [5]). A more severe challenge is represented by the time scales on which the location of the mode must be determined and reached by the EC beams before it locks [24].

The plasma parameters considered here have been taken from a time slice (close to the end of the flattop phase) of a simulated plasma discharge for the standard $I_{p}=15 \mathrm{MA}, Q=10$ ITER scenario (called Case 1 in Ref. [5]). The values $w_{\text {sat }}=32 \mathrm{~cm}$ for the $q=2$ surface and $w_{\text {sat }}=25 \mathrm{~cm}$ for the $q=3 / 2$ surface are employed, while $w_{\text {marg }}$ is treated again as a parameter. The power requirement for NTM stabilization according to a numerical solution of Eq. (6) is shown in Fig. 4 for $q=3 / 2$ parameters for $\mathrm{cw}$ injection and $w_{\text {marg }}=2 \mathrm{~cm}$. The misalignment and the $\mathrm{CD}$ profile width are normalized to the nominal profile width $w_{\mathrm{CD}, 0}$, i. e. the width obtained from standard beam tracing calculations, where profile-broadening effects are not considered. The calculation is stopped when the required power exceeds $40 \mathrm{MW}$. It can be observed that in the case of no misalignment and profile broadening by a factor of two (i. e. decrease of peak $j_{\mathrm{CD}}$ by a factor of two) the power requirements approach the range of the maximum available power from a given row of mirrors (13.3 MW, see Sec. 1).

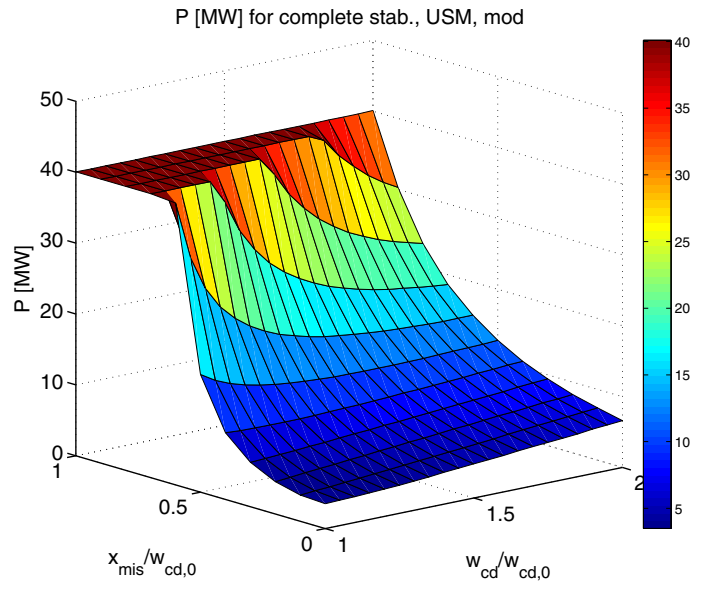

$\mathrm{P}[\mathrm{MW}]$ for complete stab., LSM, mod

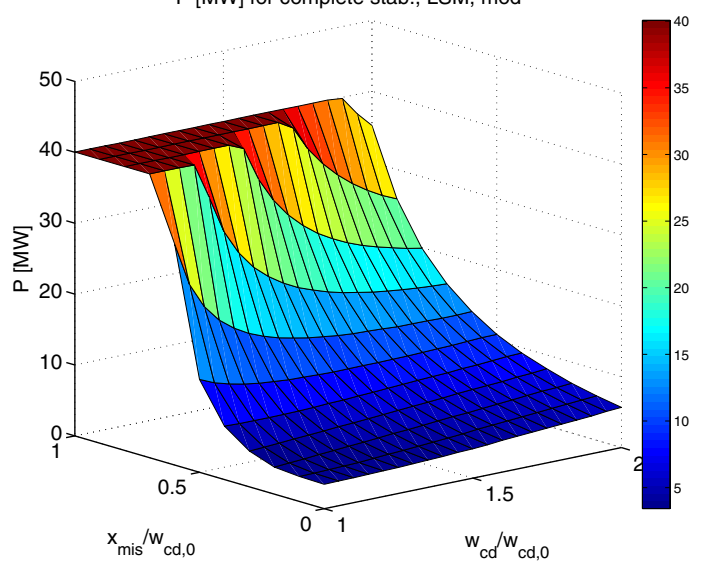

Figure 5. The same as Fig. 4 for modulated injection.

The impact of profile broadening is significantly reduced if modulated injection is considered, Fig. 5. Also the constraints on the tolerable misalignment of the beam with respect to the rational surface become less severe in this case. The power requirements can be reduced also for $\mathrm{cw}$ injection, if the aim is not full NTM suppression but rather a reduction of the magnetic island to a "tolerable" size (here we take the threshold predicted for mode locking in Ref. [25], i. e. $w=8 \mathrm{~cm}$ for the $q=3 / 2$ surface). As can be seen in Fig. 6, the impact of beam broadening is reduced also in this case, but not so strongly as in the modulated case. In the case of modulation, however, a more accurate modelling could become necessary, since the time needed for the generation of ECCD through the Fisch-Boozer mechanism could become of the same order as the island rotation time in front of the launcher [11] and the assumption of an instantaneously generated current could be poorly justified.

Similar conclusions can be drawn for the $q=2$ surface. The same calculations have been repeated for different values of $w_{\text {marg }}$. It turns out that, for $w_{\text {marg }} \geq 4 \mathrm{~cm}$, a reduction of $j_{\mathrm{CD}}$ by a factor of 0.5 would still lead to power requirements well within the $13.3 \mathrm{MW}$ available from a single row of mirrors also for the case of cw injection, while this number is exceeded for $w_{\text {marg }} \lesssim 2 \mathrm{~cm}$, as stated above. It should be stressed again that these conclusions are based on a simplified modelling, which is expected to 

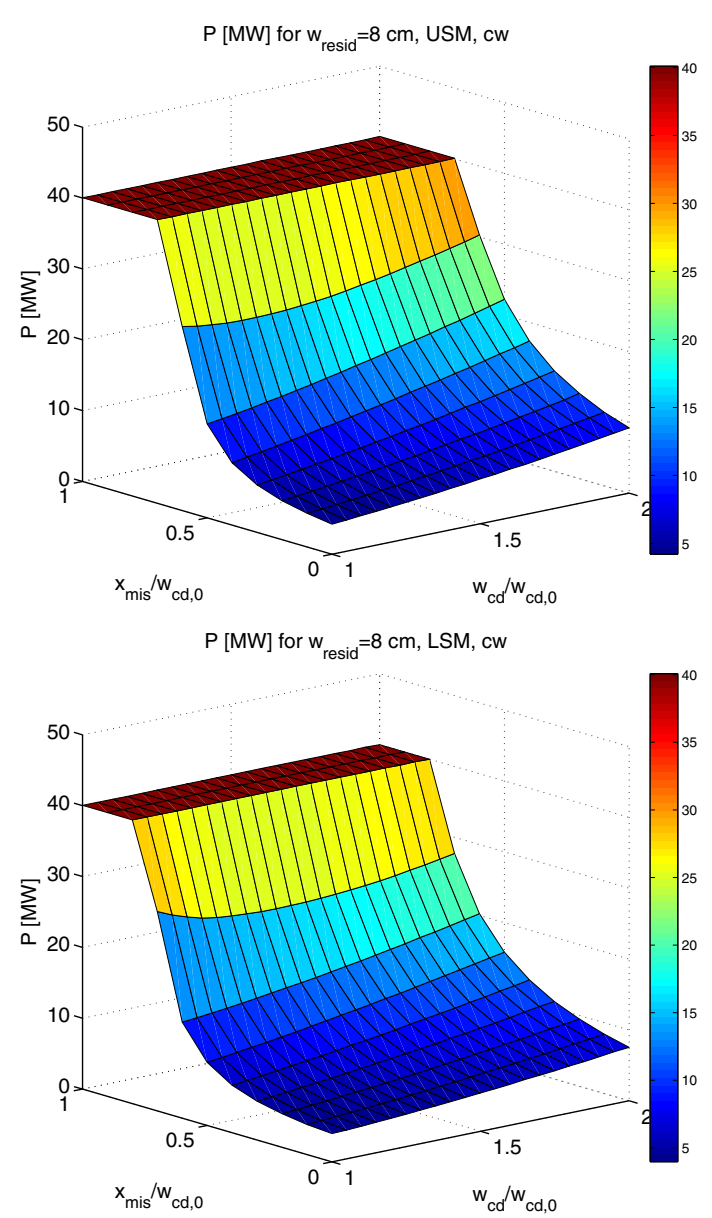

Figure 6. Power requirements for reduction of the island width below $8 \mathrm{~cm}$. Parameters again for the $q=3 / 2$ surface, cw injection and $w_{\text {marg }}=2 \mathrm{~cm}$.

reproduce correctly the physical trends, but does not aim at accurate quantitative predictions.

\section{Concluding remarks}

In this paper, a review of the NTM stabilization criteria for ITER has been presented. Their validity as sufficient conditions guiding the development of the launcher has been confirmed. From these criteria, and from beam tracing calculations performed with the present UL setup for a representative time slice of a simulated standard H-mode discharge, NTM suppression appears to be well within the capabilities of the launcher. Some physical processes that could lead to profile broadening and hence to a deviation from these results have been discussed. In particular, there is an increasing evidence that beam spreading due to density fluctuations will be significant in ITER. In case of a broadening of the profile by a factor of two, the power required for complete NTM stabilization could become marginal for $\mathrm{cw}$ injection and marginal island widths below ca. $2 \mathrm{~cm}$.

\section{References}

[1] D. Strauss, Fus. Eng. Des. 88, 2761 (2013).

[2] H. Zohm et al., Plasma Phys. Control. Fusion 49, B341 (2007).

[3] O. Sauter et al., Plasma Phys. Control. Fusion 52, 025002 (2010).

[4] N. Bertelli, D. De Lazzari, E. Westerhof, Nucl. Fusion 51103007 (2011).

[5] D. Farina, L. Figini et al., this conference.

[6] N. Bertelli, E. Westerhof, Nucl. Fusion 49095018 (2009).

[7] C. Tsironis et al., Phys. Plasmas 16112510 (2009).

[8] P. H. Rutherford, Phys. Fluids 16, 1903 (1973).

[9] O. Sauter et al., Phys. Plasmas 41654 (1997).

[10] C. C. Hegna, J. D. Callen, Phys. Plasmas 4, 2940 (1997).

[11] G. Giruzzi et al., Nucl. Fusion 39, 107 (1999).

[12] M. Kotschenreuther et al., Phys Fluids 28, 294 (1985).

[13] R. Fitzpatrick, Phys. Plasmas 2, 825 (1995).

[14] O. Sauter, Phys. Plasmas 11, 4808 (2004).

[15] R. Prater et al., Nucl. Fusion 48035006 (2008).

[16] B. Ayten, E. Westerhof, ASDEX Upgrade Team, Nucl. Fusion 54, 073001 (2014).

[17] E. Poli et al., Proc. 14th Joint Workshop on ECE and ECRH (Santorini, Greece, 2006) http://www.hellasfusion.gr/images/stories/ec14/papers/61.pdf.

[18] N. Bertelli et al., Nucl. Fusion 50115008 (2010).

[19] O. Maj, A. A. Balakin, E. Poli, Plasma Phys. Control. Fusion 52085006 (2010).

[20] Y. Peysson et al., Plasma Phys. Control. Fusion 53 124028 (2011).

[21] H. Weber, O. Maj, E. Poli, this conference.

[22] F. J. Casson et al., in preparation (2014).

[23] E. Poli, A. G. Peeters, G. V. Pereverzev, Comp. Phys. Comm. 136, 90 (2001).

[24] H. v. d. Brand et al., Plasma Phys. Control. Fusion 54, 094003 (2012).

[25] R. J. LaHaye et al., Nucl. Fusion 48, 054004 (2008). 